\title{
Apreciação da usabilidade em jogos educativos de programação voltados para crianças
}

\author{
Julia Godinho ${ }^{1}$, Gabriella Lima ${ }^{1}$, Natália Sales Santos ${ }^{2}$, Jéssica Penna ${ }^{3}$, Janaina \\ Gomide $^{1}$, Glívia Angélica R. Barbosa ${ }^{4}$ \\ ${ }^{1}$ Universidade Federal do Rio de Janeiro (UFRJ) - Campus Macaé - RJ - Brasil \\ ${ }^{2}$ Instituto de Gestão e Tecnologia da Informação (IGTI) - Belo Horizonte , MG - Brasil \\ ${ }^{3}$ Pontifícia Universidade Católica de Minas Gerais (PUC) - Belo Horizonte , MG - \\ Brasil \\ ${ }^{4}$ Centro Federal de Educação Tecnológica de Minas Gerais (CEFET) - Belo Horizonte, \\ MG - Brasil \\ \{juliagodinho08, gabriella.lima1708, nattes\}@gmail.com, \\ janainagomide@macae.ufrj.br, penna_jessica@hotmail.com, \\ gliviabarbosa@decom. cefetmg.br
}

\begin{abstract}
Usability is a quality of use that directly influences user productivity and satisfaction. One of the ways to characterize the usability of interactive systems is through the application of evaluation. This article presents the usability evaluation made on educational games, through an evaluation with users, children between 6 and 12 years, during the Summer School of Programming promoted by the extension project Aprenda a Programar Jogando from UFRJ-Macaé.

Resumo. Usabilidade é uma qualidade de uso que influencia diretamente na produtividade e satisfação dos usuários. Uma das formas de caracterizar a usabilidade de sistemas interativos é por meio da aplicação de avaliação. Este artigo apresenta a avaliação de usabilidade feita sobre os jogos educativos, por meio de uma avaliação com usuários, crianças entre 6 e 12 anos, durante a Escola de Verão de Programação promovida pelo projeto de extensão Aprenda a Programar Jogando da UFRJ - Macaé
\end{abstract}

\section{Introdução}

Jogos educativos para auxiliar no ensino e no aprendizado em programação têm sido muito utilizados. Segundo Medeiros et al. (2013) os jogos educativos têm como objetivo transformar o ato de jogar em um ato de aprender e ensinar, ajudando na construção do conhecimento.

As diferentes formas de apresentação das informações dos jogos sejam através de imagens, sons e textos, é o que atrai visualmente o usuário para a sua utilização (Calisto et al. 2010). Como os jogos influenciam e motivam a aprendizagem de programação foi percebida a necessidade de avaliar tais jogos com o objetivo de verificar se essas informações promovem uma experiência agradável ao usuário sem que o processo de aprendizado seja prejudicado. 
VII Congresso Brasileiro de Informática na Educação (CBIE 2018)

Anais do XXIX Simpósio Brasileiro de Informática na Educação (SBIE 2018)

Segundo Nielsen (1994), a usabilidade é composta por cinco dimensões: aprendizado, memorização, prevenção de erros, eficiência e satisfação. Sendo necessário analisar aquelas mais relevantes para o sistema. No contexto de jogos educativos, as dimensões mais importantes são aprendizado e satisfação. Para a realização desse trabalho dois jogos educativos foram avaliados em função da usabilidade, são eles: Monster Coding ${ }^{1}$ e Code Monkey ${ }^{2}$, ver Figura 1.
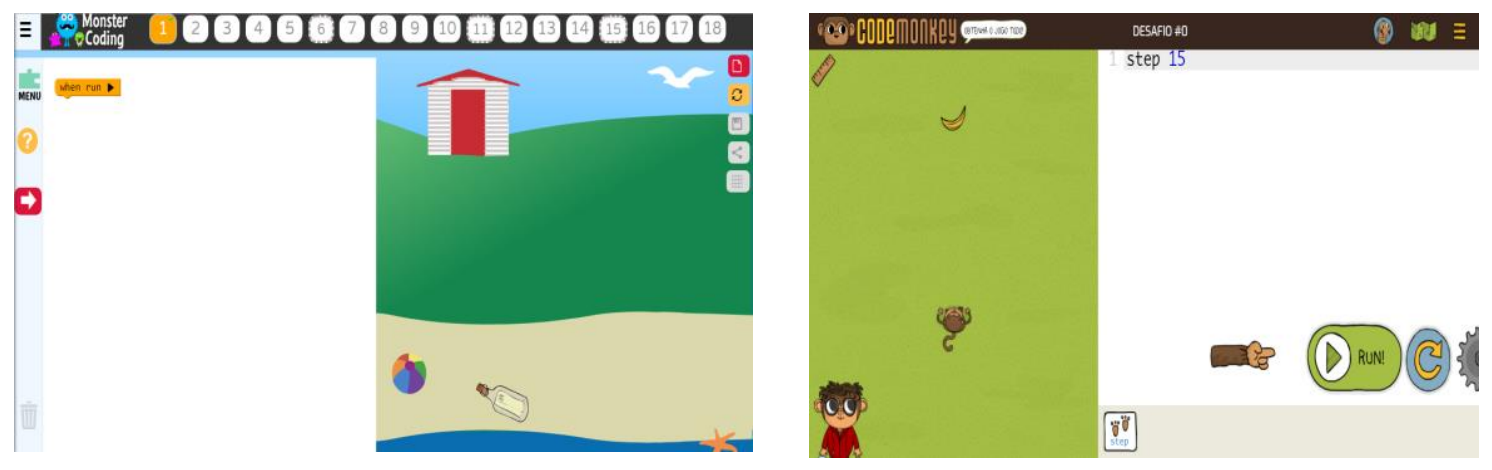

Figura 1. Monster Coding e Code Monkey

Ambos os jogos são voltados para o público infantil, não necessita de qualquer conhecimento prévio de programação e possuem diversas fases que tem o objetivo de apresentar conceitos básicos de programação aos usuários, tais como booleanos, matrizes e comandos de repetição. O Monster Coding utiliza a linguagem de programação em blocos e Code Monkey utiliza a linguagem CoffeScript, que é uma linguagem que compila JavaScript e é escrita.

\section{Metodologia}

O estudo foi realizado com crianças de 6 a 12 anos, tanto de escola pública quanto de particular, em diferentes níveis de aprendizado em programação e em inglês. Para alcançar os objetivos propostos por esse trabalho, a avaliação foi dividida em duas partes: avaliação dos usuários e questionário.

A avaliação dos usuários consistiu na observação dos mesmos durante a realização dos tutoriais dos jogos. Cada participante foi submetido à realização das tarefas com o acompanhamento de um avaliador. Foram observadas as execuções das tarefas, bem como o tempo decorrido e dificuldades encontradas pelos alunos durante o teste. As tarefas consistiam basicamente em mudar a linguagem do jogo, para o português e iniciar o tutorial do jogo. O tutorial do Moster Coding contém 18 tarefas e do Code Mokey contém 30 tarefas.

Após a avaliação um questionário foi aplicado. Esse questionário foi baseado nas heurísticas apresentadas por Barbosa et. al, (2016) nas quais foram adaptadas das dez heurísticas de Nielsen. Além das heurísticas, foram incluídas perguntas para que fosse

\footnotetext{
${ }^{1}$ Disponível em: < http://monstercoding.com/>

${ }^{2}$ Disponível em: < https://www.playcodemonkey.com/>
} 
VII Congresso Brasileiro de Informática na Educação (CBIE 2018)

Anais do XXIX Simpósio Brasileiro de Informática na Educação (SBIE 2018)

possível caracterizar o perfil dos participantes e a satisfação dos usuários a respeito dos jogos. Com o objetivo de coletar a maior quantidade de dados possível, o questionário foi aplicado aos demais alunos da Escola de Verão que não participaram da avaliação com usuários, porém realizaram as mesmas tarefas durante as aulas (totalizando 22 participações no questionário).

Como o estudo foi feito com crianças, houve uma grande preocupação com a forma como as heurísticas seriam abordadas. Por isso, foram criadas perguntas objetivas e simples para que os usuários pudessem expressar suas opiniões sobre cada aspecto dos jogos. O questionário completo (com as heurísticas e as perguntas) pode ser visto no site do projeto: https://www.aprendaprogramar.macae.ufrj.br/pesquisa.

\section{Resultados Parciais}

\subsection{Monster Coding}

A avaliação de usuários foi realizada com o total de 13 usuários com idades entre 6 e 12 anos e foi observado que as tarefas com nível de dificuldade maior tiveram maiores graus de erros, ver Figura 2. Um dos fatores determinantes para este fato foi a tradução incorreta do inglês para português e o nível ainda baixo de conhecimento em programação de alguns alunos. Foi possível perceber também que o jogo teve um índice significativo de rejeição, onde por diversas vezes os participantes reclamaram sobre falhas na tradução durante o jogo.

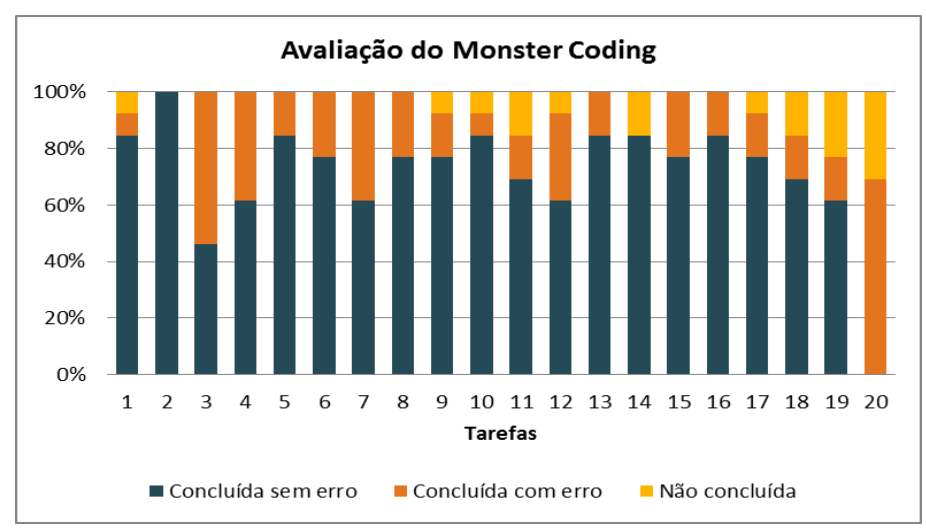

Figura 2. Grau de conclusão das tarefas

O tutorial parabeniza e incentiva constantemente o aluno a continuar jogando, em acordo com a HE2. Porém, o grande número de falhas de tradução comprometeu o entendimento dos usuários, o qual refletiu nos resultados da HE3, ver Figura 3. 
VII Congresso Brasileiro de Informática na Educação (CBIE 2018)

Anais do XXIX Simpósio Brasileiro de Informática na Educação (SBIE 2018)

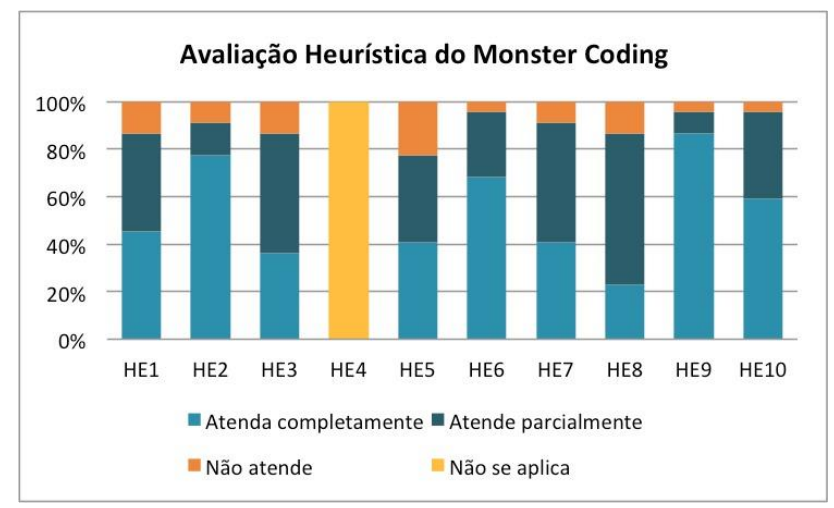

Figura 3. Incidência de adequação das heurísticas

Apesar das reclamações feitas durante o jogo, causadas pela tradução equivocada, a HE9 mostra que a maioria dos alunos se sentiu motivado durante a experiência. O questionário também mostrou que $54 \%$ dos alunos jogaria novamente e $72 \%$ indicaria o jogo a um amigo

\subsection{Code Monkey}

A avaliação de usuários foi realizada com o total de 14 usuários, com idades entre 7 e 12 anos, porém apenas seis crianças conseguiram concluir o tutorial. Dessas crianças, nove eram meninos e cinco meninas, nenhuma delas conhecia o Code Monkey. A principal reclamação dos usuários foi à quantidade de fases do jogo. De acordo com eles, o tutorial é muito extenso tornando o jogo desestimulante. Em consequência, alguns usuários não conseguiram concluir as últimas tarefas, ver Figura 4. Foi percebido também que mesmo com um tutorial extenso os usuários conseguiram aprender mais facilmente os conceitos de programação.

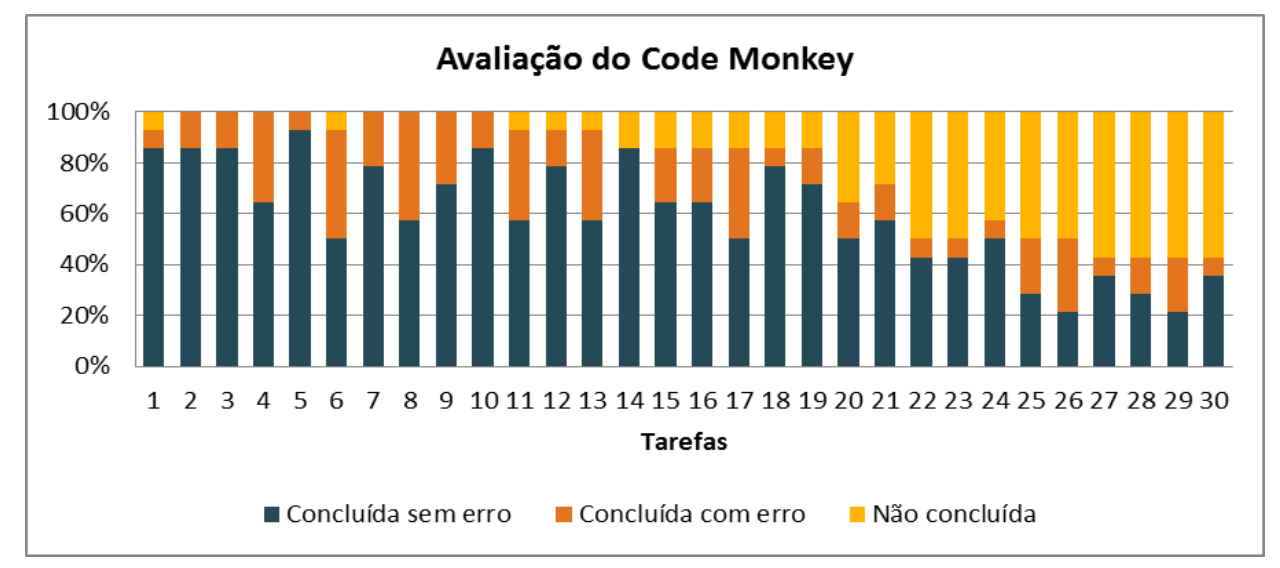

Figura 4. Grau de conclusão das tarefas

Pelo questionário, foi possível observar, que pela perspectiva das crianças esse é um tutorial com grau elevado de dificuldade HE7, ver Figura 5. Entretanto, houve a aceitação do grupo, como pode ser visto pela HE9. Além do mais, foi percebido pelos avaliadores que mesmo com um tutorial extenso, os usuários conseguiram aprender mais facilmente os conceitos de programação conforme HE5 e HE6. Além disso, o 
VII Congresso Brasileiro de Informática na Educação (CBIE 2018)

Anais do XXIX Simpósio Brasileiro de Informática na Educação (SBIE 2018)

questionário confirmou a aceitação do jogo, dado que mais de $80 \%$ disse que indicaria o jogo para um amigo e $46 \%$ disse que jogaria novamente.

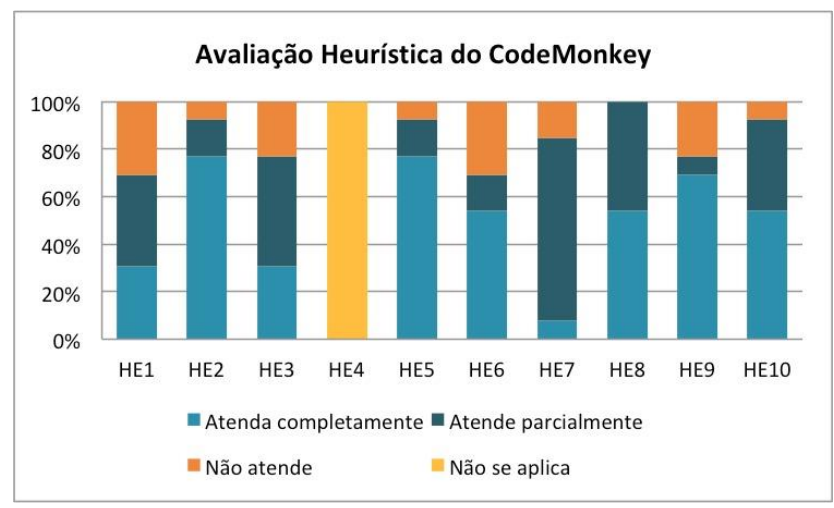

Figura 5. Incidência de adequação das heurísticas

\section{Considerações Finais}

A análise estudos de jogos educativos, como o apresentado, motiva o desenvolvimento e melhoria de jogos, de forma que cumpram seu objetivo na aprendizagem da programação de forma mais efetiva, fazendo diagnóstico de possíveis causas de erro de interpretação e/ou código.

Os resultados parciais nos encorajam a continuar com a avalição dos jogos educativos, não só na perspectiva dos usuários, mas também da perspectiva de especialistas em usabilidade. Com essa avaliação será possível verificar se os potenciais problemas listados pelos especialistas, de fato impactam os usuários durante a utilização do jogo.

Os próximos passos envolvem a realização de avaliações com educadores que utilizam jogos educativos no ensino de programação a fim de contrastar os problemas encontrados pelos educadores em nível de ensino com os problemas encontrados pelos usuários em nível de aprendizado.

\section{Referências}

Barbosa, G., Oliveira, E., \& D'Carlo, D. (2016). Usabilidade em aplicativos móveis educacionais: Um conjunto de heurísticas para avaliação. In Simpósio Brasileiro de Informática na Educação-SBIE (Vol. 27, No. 1, p. 777).

Calisto, A., Barbosa, D., \& Silva, C. (2010). Uma análise comparativa entre jogos educativos visando a criação de um jogo para educação ambiental. In Simpósio Brasileiro de Informática na Educação-SBIE (Vol. 1, No. 1).

Medeiros, T. J., da Silva, T. R., \& da Silva Aranha, E. H. (2013). Ensino de programação utilizando jogos digitais: uma revisão sistemática da literatura. RENOTE, 11(3).

Nielsen, J. (1994). Usability engineering. Elsevie 